\title{
Epidemiologia dos tumores do sistema nervoso simpático na população infanto- juvenil em Sergipe: uma análise de 18 anos
}

\author{
Epidemiology of sympathetic nervous system tumors in the childhood population in Sergipe: an 18- \\ years analysis
}

Epidemiología de los tumores del sistema nervioso simpático en la población Infantil y Juvenil de Sergipe: un análisis de 18 años

Recebido: 30/08/2021 | Revisado: 07/09/2021 | Aceito: 07/09/2021 | Publicado: 09/09/2021

\author{
Milenna Guimarães Lima \\ ORCID: https://orcid.org/0000-0002-8838-1690 \\ Universidade Tiradentes, Brasil \\ E-mail: milennaguimaraesl@gmail.com \\ Ana Jovina Barreto Bispo \\ ORCID: https://orcid.org/0000-0002-6228-768X \\ Universidade Tiradentes, Brasil \\ E-mail: anajovina70@gmail.com \\ Mariana Aragão Passos \\ ORCID: https://orcid.org/0000-0003-3855-8379 \\ Universidade Tiradentes, Brasil \\ E-mail: mariana_aragaopassos@hotmail.com
}

\begin{abstract}
Resumo
Objetivo: Descrever a epidemiologia dos Tumores do Sistema Nervoso Simpático no Estado de Sergipe ao longo de 18 anos. Métodos: Trata-se de um estudo transversal, retrospectivo, quantitativo. Os dados foram coletados no Registro de Câncer de Base Populacional (RCBP) do Centro de Oncologia Pediátrica localizado no Hospital de referência do Estado de Sergipe. Foram incluídos todos os pacientes com idades entre 0 e 19 anos constantes no RCBP no período de 1996 a 2014. Resultados: Em Sergipe, no período de 1996-2014 foram registrados 1705 casos de Cânceres Infanto-Juvenis, destes 45 (2,6\%) eram tumores do Sistema Nervoso Simpático. Predominaram pacientes do gênero masculino (53,3\%) e faixa etária mais acometida foi a de menores de 5 anos $(71,1 \%)$. Observou-se uma taxa de crescimento percentual anual ao longo desse tempo de $0,08 \%$. Conclusão: Percebeu-se que a incidência dos TSNS ainda é baixa em relação a outros estados do Brasil e a outros países com mais recursos, o que pode evidenciar a importância de melhorar o diagnóstico dos tumores do sistema nervoso simpático em Sergipe.
\end{abstract}

Palavras-chave: Neoplasia; Epidemiologia; Neuroblastoma; Criança; Adolescente.

\begin{abstract}
Objective: To describe the epidemiology of Sympathetic Nervous System Tumors in the State of Sergipe over 18 years. Methods: This is a cross-sectional, retrospective, quantitative study. Data were collected from the Population-Based Cancer Registry of the Pediatric Oncology Center located at the Sergipe State Reference Hospital. All patients aged between 0 and 19 years listed in the RCBP from 1996 to 2014 were included. Results: In Sergipe, between 1996 and 2014, 1705 cases of Childhood Cancer were registered, of which 45 (2.6\%) were tumors of the Sympathetic Nervous System. There was a predominance of male patients $(53.3 \%)$ and the most affected group were those under 5 years old $(71.1 \%)$. An annual percentage growth rate of $0.08 \%$ over this period was observed. Conclusion: It was noticed that the incidence of TSNS is still low compared to other states in Brazil and other countries with more resources, which can highlight the importance of improving the diagnosis of tumors of the sympathetic nervous system in Sergipe.
\end{abstract}

Keywords: Neoplasm; Epidemiology; Neuroblastoma; Child; Adolescent.

\section{Resumen}

Objetivo: Describir la epidemiología de los tumores del sistema nervioso simpático en el estado de Sergipe durante 18 años. Métodos: Estudio transversal, retrospectivo y cuantitativo. Los datos se obtuvieron del Registro de cáncer de base poblacional (RCBP) del Centro de Oncología Pediátrica ubicado en el Hospital Estatal de Referencia de Sergipe. Se incluyeron todos los pacientes con edades comprendidas entre 0 y 19 años incluidos en el RCBP de 1996 a 2014. Resultados: En Sergipe, en el período 1996-2014 se registraron 1705 casos de Cáncer Infantil y Juvenil, de los cuales $45(2,6 \%)$ fueron tumores del sistema nervioso simpático. Predominó el sexo masculino $(53,3 \%)$ y el grupo de edad más afectado fue el de los menores de 5 años $(71,1 \%)$. Se observó una tasa de crecimiento porcentual anual de $0.08 \%$ 
durante este período. Conclusión: Se notó que la incidencia de TSNS aún es baja en comparación con otros estados de Brasil y otros países con más recursos, lo que puede resaltar la importancia de mejorar el diagnóstico de tumores del sistema nervioso simpático en Sergipe.

Palabras clave: Neoplasia; Epidemiología; Neuroblastoma; Niño; Adolescente.

\section{Introdução}

Os Tumores do Sistema Nervoso Simpático (TSNS) surgem na medula adrenal ou nos gânglios paraespinhais e se manifestam mais comumente na cavidade abdominal, podendo se desenvolver também em locais como região torácica, pélvica e cervical. São representados pelo Neuroblastoma (NB), Ganglioneuroblastoma e Ganglioneuroma. O neuroblastoma e o ganglioneuroblastoma são tumores malignos, enquanto o ganglioneuroma é um tumor benigno. O tipo mais estudado é o NB pela sua incidência maior (Behera et al, 2020).

O NB é um tumor embrionário do sistema nervoso autônomo e sua origem está nas células da crista neural (Maris et al, 2010), que servem como células-tronco multipotentes que se diferenciam em tecidos neurais periféricos maduros (Nakagawara et al, 2018). A probabilidade de desenvolver NB varia conforme a idade, sendo que $96 \%$ dos casos ocorrem em crianças menores de 10 anos (Chang et al, 2017). É raro que o NB se manifeste em adolescentes e adultos, e quando isso ocorre, a doença tende a ser mais letal (Nakagawara et al, 2018).

A doença então pode variar de uma massa indolor detectada acidentalmente a um tumor de crescimento rapidamente progressivo, amplamente disseminado. As manifestações clínicas mais frequentemente associadas a tumoração são febre, dor abdominal e dor óssea (Lucena et al, 2018). Alguns pacientes podem apresentar sintomas específicos como a Síndrome de Horner, que é causada pela compressão da medula espinhal provocada pela agressividade local do tumor ou por infiltração de lesões primárias no tecido neural (Nakagawara et al, 2018).

A tomografia computadorizada (TC) e a ressonância magnética (RM) são recomendadas para o diagnóstico e permitem avaliar as características do tumor, assim como realizar o estadiamento da doença (Burnand et al, 2019; Brisse et al, 2017). O tratamento dos pacientes envolve quimioterapia e cirurgia (Ahmed et al, Coughlan et al, 2017). O prognóstico da doença é variável, podendo alguns tumores sofrerem regressão espontânea, outros serem tratados apenas com cirurgia ou após redução com quimioterapia, enquanto alguns ainda podem exibir comportamento agressivo e progressão refratária ao tratamento (Liu et al, 2020; Yang et al, 2020; Amano et al, 2018). A sobrevida dos pacientes de alto risco apresenta taxas em torno de 50\% (SU et al, 2019).

O presente estudo tem como objetivo descrever a epidemiologia dos Tumores do Sistema Nervoso Simpático no estado de Sergipe ao longo de 18 anos, de 1996 até 2014. Nota-se que ainda são escassas as publicações com dados acerca da epidemiologia dos Cânceres Infanto-Juvenis no Brasil, principalmente de tipos específicos, como é abordado nesse artigo. Assim, justifica-se a relevância desse estudo, principalmente por abranger um período considerável.

\section{Metodologia}

Trata-se de um estudo transversal, retrospectivo, quantitativo. Os dados foram coletados no Registro de Câncer de Base Populacional (RCBP) do Centro de Oncologia Pediátrica localizado no Hospital de referência do Estado de Sergipe. Foram incluídos todos os pacientes com idades entre 0 e 19 anos constantes no RCBP no período de 1996 a 2014.

Os dados foram descritos por meio de frequência absoluta e relativa. A hipótese de estabilidade das medidas de tendência central ao longo dos anos foi testada por meio do teste de Kruskal-Wallis.

A taxa de crescimento anual percentual foi quantificada utilizando o programa Jointpoint Regression Program (Versão 4.5.0.1). As demais análises foram feitas utilizando o software R Core Team 2021 (Versão 4.0.4) e o nível de significância adotado foi de $5 \%$. 
Este estudo foi submetido e aprovado pelo Comitê de Ética em Pesquisa da Universidade Tiradentes, sob o parecer $n^{\circ}$ 3.239.059.

\section{Resultados}

Em Sergipe, do ano de 1996 até o ano de 2014, foram registrados, no banco de dados do Centro de Oncologia do Hospital analisado, 1705 casos de câncer infanto-juvenil. Os tumores do Sistema Nervoso Simpático corresponderam a 2,6\% do total de casos (Tabela 1).

Tabela 1. Frequência dos cânceres infanto-juvenis no estado de Sergipe, durante o período de 1996-2014.

\begin{tabular}{|c|c|c|}
\hline $\begin{array}{l}\text { CLASSIFICAÇÃO INTERNACIONAL DO CÂNCER INFANTO- } \\
\text { JUVENIL }\end{array}$ & $\mathbf{N}$ & $\%$ \\
\hline I (Leucemias) & 376 & 22,0 \\
\hline $\begin{array}{l}\text { II (Linfomas Neoplasias } \\
\text { Reticuloendoteliais) }\end{array}$ & 304 & 17,8 \\
\hline III (Neoplasias do SNC) & 242 & 14,1 \\
\hline $\begin{array}{l}\text { IV (Tumores do Sistema Nervoso } \\
\text { Simpático) }\end{array}$ & 45 & 2,6 \\
\hline V (Retinoblastoma) & 26 & 1,5 \\
\hline VI (Tumores Renais) & 69 & 4,0 \\
\hline VII (Tumores Hepáticos) & 23 & 1,3 \\
\hline VIII (Tumores ósseos malignos) & 94 & 5,5 \\
\hline IX (Sarcoma de partes moles) & 99 & 5,8 \\
\hline $\mathrm{X}$ (Neoplasias de células germinativas) & 55 & 3,2 \\
\hline XI (Outras neoplasias epiteliais malignas) & 292 & 17,1 \\
\hline $\begin{array}{l}\text { XII (Outras neoplasias malignas } \\
\text { inespecíficas) }\end{array}$ & 80 & 4,6 \\
\hline TOTAL & 1705 & 100 \\
\hline
\end{tabular}

Fonte: Dados da pesquisa (2020).

Na Tabela 2, verifica-se que a prevalência de casos dos Tumores do Sistema Nervoso Simpático (IV) ao longo dos anos se manteve constante, com taxa de crescimento anual percentual pouco significativa $(0,08 \%)$ (Tabela 2$)$. 
Tabela 2. Taxa de crescimento anual dos casos de câncer infanto-juvenil no Estado de Sergipe de 1996 a 2014

\begin{tabular}{|c|c|c|c|c|}
\hline & \multicolumn{4}{|c|}{ IC $95 \%$} \\
\hline & TCAP & - & + & p-valor \\
\hline \multicolumn{5}{|l|}{ Sexo } \\
\hline Feminino & $-1,164$ & $-2,875$ & 0,577 & 0,175 \\
\hline Masculino & 1,754 & 0,242 & 3,289 & 0,025 \\
\hline \multicolumn{5}{|l|}{ CICI } \\
\hline I & 0,621 & $-1,772$ & 3,074 & 0,594 \\
\hline II & $-0,487$ & $-2,715$ & 1,792 & 0,655 \\
\hline III & $-0,024$ & $-3,042$ & 3,088 & 0,987 \\
\hline IV & 0,080 & $-3,847$ & 4,167 & 0,967 \\
\hline $\mathrm{V}$ & $-6,747$ & $-10,812$ & $-2,497$ & 0,004 \\
\hline VI & $-3,520$ & $-7,047$ & 0,140 & 0,058 \\
\hline VII & $-1,658$ & $-8,491$ & 5,685 & 0,630 \\
\hline VIII & $-1,222$ & $-5,272$ & 3,001 & 0,544 \\
\hline IX & $-4,421$ & $-8,348$ & $-0,324$ & 0,036 \\
\hline$X$ & $-2,393$ & $-6,838$ & 2,263 & 0,288 \\
\hline XI & 4,498 & 1,624 & 7,453 & 0,004 \\
\hline XII & 0,909 & $-8,487$ & 11,271 & 0,847 \\
\hline
\end{tabular}

Legenda: TCAP- Taxa de crescimento anual percentual. IC95\%- Intervalo com 95\% de confiança. Fonte: Dados da pesquisa (2020)

Durante o intervalo de tempo analisado, os TSNS acometeram mais o gênero masculino (53,3\%). A incidência foi decrescente de acordo com a idade, sendo a faixa etária mais acometida a de menores de 5 anos (71,1\%), seguida de 5-9 anos (13,3\%), 10-14 anos (8,8\%) e 15-19 anos (6,6\%). O período com maior número de casos registrados foi o de 2009-2014 (37,7\%), e o período com menor registro foi o de 1996-2001 (26,6\%) (Tabela 3).

Tabela 3. Incidência dos Tumores do Sistema Nervoso Simpático, em relação aos anos de ocorrência, gêneros e faixa etária.

\begin{tabular}{lll}
\hline ANOS & $\mathbf{N}$ & $\%$ \\
\hline $1996-2001$ & 12 & 26,6 \\
$2002-2008$ & 16 & 35,5 \\
$2009-2012$ & 17 & 37,7 \\
\hline GÊNERO & & 46,6 \\
\hline FEMININO & 21 & 53,3 \\
MASCULINO & 24 & \\
\hline FAIXA ETÁRIA & & 71,1 \\
\hline 0-4 anos & 32 & 13,3 \\
5-9 anos & 6 & 8,8 \\
$10-14$ anos & 4 & 6,6 \\
\hline $15-19$ anos & 3 & \\
\hline
\end{tabular}

Fonte: Dados da pesquisa (2020). 
Em relação a idade do diagnóstico, os menores de 5 anos foram os mais acometidos em todos os anos analisados, com um aumento no percentual ao longo dos anos (1996-2001: 66\%, 2002-2008: 68,75\% e de 2009-2014: 76,4\%).

No decorrer do tempo, verificou-se uma frequência semelhante do total de cânceres infanto-juvenis entre os gêneros, com um leve predomínio do sexo feminino (50,5\%). No entanto, ao analisar cada grupo, percebe-se que linfomas e neoplasias reticuloendoteliais $(66,7 \%)$, retinoblastoma $(61,5 \%)$ e tumores hepáticos $(60,8 \%)$ têm um predomínio entre crianças e adolescentes do sexo feminino, enquanto em neoplasias de células germinativas, outras neoplasias malignas e tumores renais o percentual de sexo masculino é consideravelmente maior que o feminino, com 78,1\%, 73,3\% e 55\%, respectivamente (Gráfico 1).

Gráfico 1. Distribuição por gênero dos casos de câncer infanto-juvenil no Estado de Sergipe de 1996 a 2014.

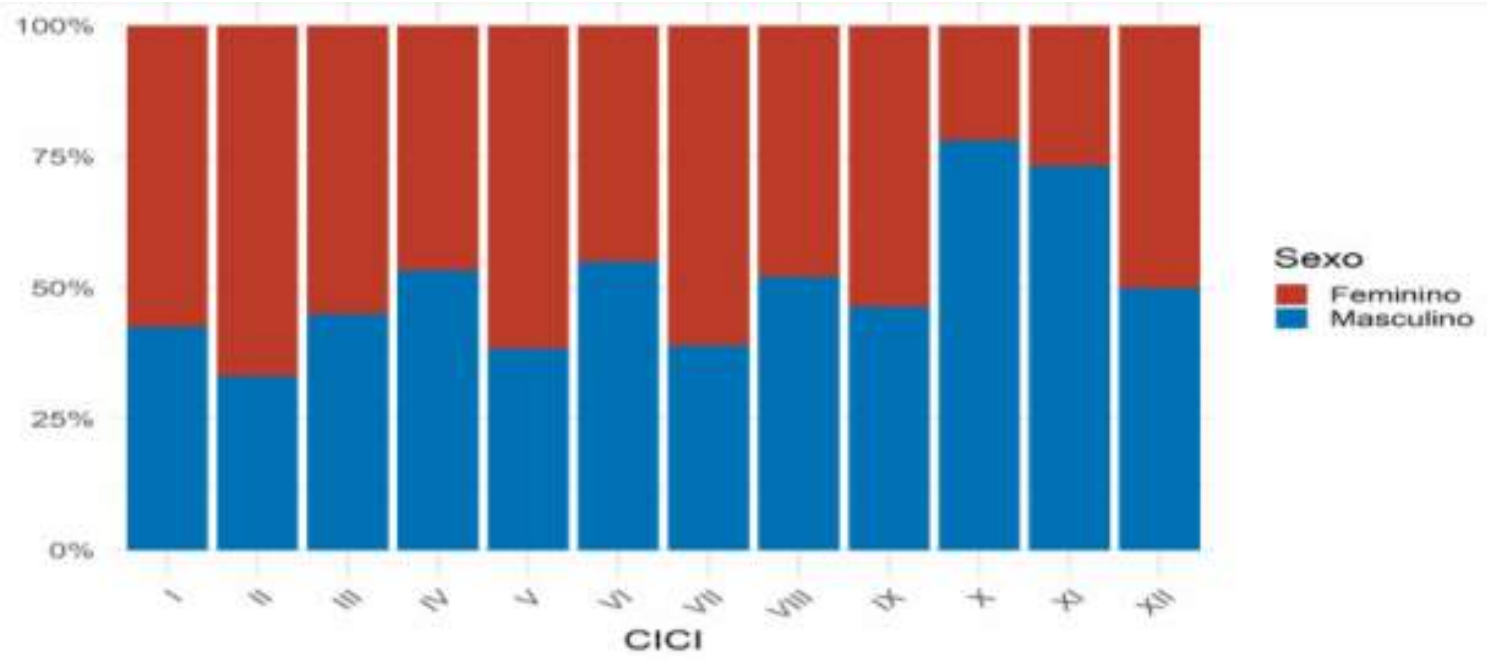

Fonte: Dados da pesquisa (2020).

Como pode ser observado no Gráfico 2, a idade no momento do diagnóstico dos CIJ aumentou no passar dos anos estudados. Em 1996, a idade média do diagnóstico foi de 8,9 anos, enquanto em 2014 foi de 11,4 anos (Gráfico 2).

Gráfico 2. Idades de diagnóstico de câncer infanto-juvenil no Estado de Sergipe de 1996 a 2014

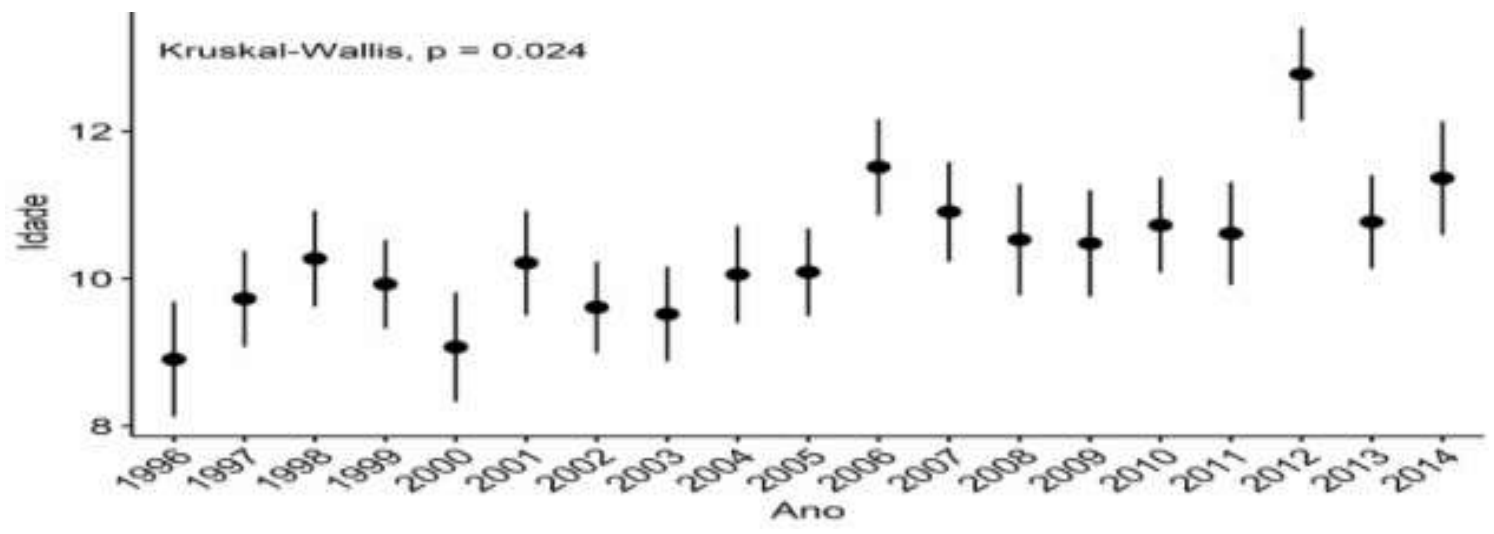

Fonte: Dados da pesquisa (2020). 


\section{Discussão}

O presente estudo revelou que os Tumores do Sistema Nervoso Simpático, representados principalmente pelo Neuroblastoma, corresponderam a 2,6\% de todos os casos de cânceres infanto-juvenis em Sergipe, durante o período de 19962004. Santos et al, em um estudo realizado em 2018, no estado de Alagoas, relatou 5,1\% de TSNS entre casos de cânceres infanto-juvenis. Uma análise realizada por Camargo et al (2011) em 12 cidades brasileiras e no Distrito Federal relatou a incidência de 5,9 casos por milhão de habitantes, sendo a menor taxa em Manaus, com 2,3 casos e a maior em Curitiba, com 14,2 casos. As Regiões com melhores condições de vida relataram uma incidência maior desse tipo de câncer.

Em Sergipe, a incidência dos TSNS foi menor do que as encontradas em outros estados. Como acredita-se que exista uma maior precisão diagnóstica nas regiões com melhores condições de vida e de acesso à saúde, acreditamos que, em Sergipe, pode haver um subdiagnóstico. Ao mesmo tempo, a forma de apresentação do NB pode apresentar uma heterogeneidade clínica muito ampla, e geralmente essas variações estão relacionadas a fatores como idade do paciente, localização do tumor, estágio, presença de metástase e de síndromes paraneoplásicas (Lucena et al, 2018).

Conforme visto, ao longo do tempo, uma Taxa de Crescimento Anual dos TSNS muito pouco significativa, não correspondendo ao crescimento populacional, como visto na grande maioria dos outros cânceres infanto-juvenis. A maior precisão no diagnóstico através de novas tecnologias, o aumento da utilização dos cuidados de saúde, a melhoria da notificação do câncer e possíveis fatores ambientes são razões apontadas para o aumento dos cânceres infanto juvenis (Heck et al, 2020; Ward et al, 2014). Nos Estados Unidos, em uma pesquisa realizada por Ward et al (2014), entre 1975 e 2010 , o aumento da incidência variou entre $0,7 \%$ e $1,2 \%$ ao ano, com taxa de crescimento anual em torno de $0,6 \%$. A insignificante taxa de crescimento anual verificada no presente estudo é motivo de preocupação por, provavelmente, estar relacionada ao subdiagnóstico, seja por dificuldade de acesso aos serviços de saúde e aos meios diagnósticos ou por condução médica inadequada, resultando em um desfecho desfavorável em certa proporção dos casos antes do diagnóstico final.

No Japão, durante 20 anos foi realizado um programa de rastreamento em massa, que mostrou que a incidência de NB se tornou quase o dobro após o início do programa, sem uma mudança significativa na mortalidade geral, sugerindo a possibilidade de melhora do prognóstico paralela à aumento do diagnóstico (Nakagawara et al, 2018).

O diagnóstico do câncer depende da busca por serviço médico no início dos sintomas e da perspicácia e sabedoria do médico em considerar a possibilidade de câncer, estabelecendo o pronto diagnóstico e adequado encaminhamento (INCA, 2008). Como os sinais e sintomas do NB não são específicos e podem demorar a aparecer, muitas vezes fazer o diagnóstico precoce é difícil para os pediatras (Lucena et al, 2018). Além disso, pacientes podem apresentar regressão espontânea, sobretudo os menores de 18 meses, o que pode aumentar ainda mais o subdiagnóstico, levando em consideração que o NB de baixo risco nessa população pode se manifestar com poucos sintomas e regredir sem que seja diagnosticado (Yang et al, 2020).

O gênero mais acometido pelos Tumores do Sistema Nervoso Simpático foi o masculino (53,3\%), concordando com os resultados de Nakagawara et al (2018), Alvi e Sameer et al (2017), Lucena et al (2018). Já na análise do INCA (2008), em um estudo realizado de 1996-2000 em Aracaju, o sexo predominante foi o feminino, o que diverge do restante da literatura. Estudo de Shuangshoti, et al (2012) realizado no Sudeste da Ásia, mostrou em uma coorte 39 pacientes do sexo masculino e 23 pacientes do sexo feminino com neuroblastoma. Ward, et al (2014) relataram que em crianças houve predomínio de incidência nos meninos. Já em adolescentes, esse estudo mostrou que as taxas de incidência são semelhantes entre meninos e meninas. NB é uma doença mais comum em meninos do que nas meninas, no entanto, a base genética e epigenética para essa preponderância ainda permanece incerta (Nakagawara et al, 2018). 
O diagnóstico dos TSNS predominou em menores de cinco anos, corroborando com os achados de Lucena et al (2018), de Alvi et al (2017) e de Shuangshoti et al (2012). Em uma análise realizada pelo INCA em Aracaju, capital do estado de Sergipe, os TSNS acometeram mais a faixa etária de 0 a 4 anos (37,77\%). Como se trata de uma doença proveniente de tecidos em desenvolvimento, os tecidos da crista neural, ela se desenvolve nos primeiros anos de vida, possibilitando diagnóstico em lactentes (Yang et al; Liu et al, 2020).

Quando levamos em consideração o conjunto de Cânceres Infanto-Juvenis, no nosso estudo houve um predomínio do sexo feminino (50,5\%), resultado divergente do que foi encontrado por Santos et al (2018), no estado de Alagoas, no período de 2013 a 2016, em que os acometidos eram do sexo masculino em sua maioria (58\%), assim como no estudo de Camargo et al (2010), que analisou os registros de câncer de base populacional no Brasil, e teve predomínio do sexo masculino, exceto em Fortaleza e Jaú, que, assim como no nosso estudo, o predomínio também foi do sexo feminino. Em pacientes avaliados entre 0 a 19 anos, a faixa etária mais acometida nessa pesquisa foi entre de 15-19 anos, com 36,8\%, enquanto o estudo de Santos et al (2018) mostrou predomínio em pacientes de 5 a 9 anos com 27,7\% e o estudo de Camargo et al (2010), mostrou maior acometimento de crianças entre 1 a 4 anos.

\section{Conclusão}

Então, percebemos que, em Sergipe, os TSNS corresponderam a 2,6\% de todos os CIJ, entre 1996-2014, com prevalência constante ao longo dos anos, sem taxa de crescimento anual significativa. $O$ gênero mais acometido por esse tipo de câncer foi o sexo masculino e a faixa etária mais prevalente foi a que abrange os menores de 5 anos.

O presente estudo assume importância por estudar um período longo e revelar que a incidência no Estado de Sergipe ainda é baixa em relação a outros estados do Brasil e a outros países com mais recursos de saúde, o que nos leva a crer que é preciso melhorar o diagnóstico dos TSNS no Estado de Sergipe. O pediatra é o primeiro profissional que os cuidadores buscam diante de problemas de saúde nas crianças. Esse profissional deve estar capacitado e atento aos sinais e sintomas do NB e precisam conhecer os serviços de referência para o pronto encaminhamento diante da suspeita diagnóstica.

As informações geradas pelo RCBP são essenciais para o planejamento da rede de atenção e para assegurar o acesso oportuno. O RCBP realiza busca ativa de casos nas fontes notificadoras. Faz-se necessário o registro adequado das fontes notificadoras para um registro fidedigno da situação de cada Estado, o que podemos considerar como limitação desse estudo.

Sugerimos que trabalhos futuros abordem a epidemiologia de outras localidades, ou do próprio estado de Sergipe em outros períodos, para comparar a evolução do diagnóstico e a incidencia da doença em outros estados do Brasil ao longo do tempo.

\section{Agradecimentos}

Os autores agradecem especialmente ao Dr. Walter Marcelo Carvalho (in memorian) pela valorosa contribuição na coleta e registro dos dados fornecidos para o estudo e por todo incentivo na elaboração do projeto.

\section{Referências}

Ahmed, A. A., Zhang, L., Reddivalla, N., \& Hetherington, M. (2017). Neuroblastoma in children: update on clinicopathologic and genetic prognostic factors. Pediatric hematology and oncology, 34(3), 165-185.

Alvi, S., Karadaghy, O., Manalang, M., \& Weatherly, R. (2017). Clinical manifestations of neuroblastoma with head and neck involvement in children. International journal of pediatric otorhinolaryngology, 97, 157-162.

Amano, H., Uchida, H., Tanaka, Y., Tainaka, T., Mori, M., Oguma, E., \& Koh, K. (2018). Excellent prognosis of patients with intermediate-risk neuroblastoma and residual tumor postchemotherapy. Journal of pediatric surgery, 53(9), 1761-1765. 
Barros Rodrigues, M., Chong-Silva, D. C., \& Neto, H. J. C (2021). Guia Prático para Produção e Submissão de Artigos Científicos.

Behera, G., Chhabra, G., Mishra, P., \& Sable, M. (2020). Pediatric neuroblastic tumors: A critical evaluation of cytomorphological features for risk stratification on aspiration cytology. Diagnostic cytopathology, 48(5), 464-474.

Brisse, H. J., Blanc, T., Schleiermacher, G., Mosseri, V., Philippe-Chomette, P., Janoueix-Lerosey, I., \& Sarnacki, S. (2017). Radiogenomics of neuroblastomas: relationships between imaging phenotypes, tumor genomic profile and survival. PloS one, 12(9), e0185190.

Burnand, K., Barone, G., McHugh, K., \& Cross, K. (2019). Preoperative computed tomography scanning for abdominal neuroblastomas is superior to magnetic resonance imaging for safe surgical planning. Pediatric blood \& cancer, 66(11), e27955.

Camargo, B., de Oliveira Santos, M., Rebelo, M. S., de Souza Reis, R., Ferman, S., Noronha, C. P., \& Pombo-de-Oliveira, M. S. (2010). Cancer incidence among children and adolescents in Brazil: First report of 14 population-based cancer registries. International journal of cancer, 126(3), 715-720.

Chang, H. H., Liu, Y. L., Lu, M. Y., Jou, S. T., Yang, Y. L., Lin, D. T., \& Hsu, W. M. (2017). A multidisciplinary team care approach improves outcomes in high-risk pediatric neuroblastoma patients. Oncotarget, 8(3), 4360.

Coughlan, D., Gianferante, M., Lynch, C. F., Stevens, J. L., \& Harlan, L. C. (2017). Treatment and survival of childhood neuroblastoma: evidence from a population-based study in the United States. Pediatric hematology and oncology, 34(5), 320-330.

Estrela, C. (2018). Metodologia Científica: Ciência, Ensino, Pesquisa. Editora Artes Médicas.

Heck, J. E., Lee, P. C., Wu, C. K., Tsai, H. Y., Ritz, B., Arah, O. A., \& Li, C. Y. (2020). Gestational risk factors and childhood cancers: A cohort study in Taiwan. International journal of cancer, 147(5), 1343-1353.

INCA. Instituto Nacional de Câncer (2008). Câncer na criança e no adolescente no Brasil: dados dos registros de base populacional e de mortalidade.

Liu, Q., Feng, L., Xue, H., Su, W., \& Li, G. (2020). Development and validation of a nomogram to predict the overall survival of patients with neuroblastoma. Medicine, 99(10).

Lucena, J. N., Alves, M. T. S., Abib, S. C. V., Souza, G. O. D., Neves, R. P. D. C., \& Caran, E. M. M. (2018). Clinical and epidemiological characteristics and survival outcomes of children with neuroblastoma: 21 years of experience at the Instituto de Oncologia Pediátrica, in São Paulo, Brazil. Revista Paulista de Pediatria, 36, 254-260.

Maris, J. M. (2010). Recent advances in neuroblastoma. New England Journal of Medicine, 362(23), 2202-2211.

Nakagawara, A., Li, Y., Izumi, H., Muramori, K., Inada, H., \& Nishi, M. (2018). Neuroblastoma. Japanese journal of clinical oncology, 48(3), 214-241. https://doi.org/10.1093/jjco/hyx176.

Santos Júnior, C. J., Romão, C. M. D. S. B., Alves, M. J. R. G., Batinga, A. M. C. S., da Silva Gomes, V. M., Araújo, N. S., \& Leite, L. A. C. (2018). Características clínico-epidemiológicas do câncer infantojuvenil no estado de Alagoas, Brasil. Revista de Medicina, 97(5), 454-460.

Su, Y., Wang, L., Wang, X., Yue, Z., Xing, T., Zhao, W., \& Ma, X. (2019). Dynamic alterations of plasma cell free DNA in response to chemotherapy in children with neuroblastoma. Cancer medicine, 8(4), 1558-1566.

Shuangshoti, S., Shuangshoti, S., Nuchprayoon, I., Kanjanapongkul, S., Marrano, P., Irwin, M. S., \& Thorner, P. S. (2012). Natural course of low risk neuroblastoma. Pediatric blood \& cancer, 58(5), 690-694.

Ward, E., DeSantis, C., Robbins, A., Kohler, B., \& Jemal, A. (2014). Childhood and adolescent cancer statistics, 2014. CA: a cancer journal for clinicians, 64(2), 83-103.

Yang, C. L., Serra-Roma, A., Gualandi, M., Bodmer, N., Niggli, F., Schulte, J. H., \& Shakhova, O. (2020). Lineage-restricted sympathoadrenal progenitors confer neuroblastoma origin and its tumorigenicity. Oncotarget, 11(24), 2357. 